\title{
Correlation Between Erythrocyte Malondialdehye Levels and Dyslipidemia in Patients with Type 2 Diabetes Mellitus
}

\author{
Basavaraj Savadi ${ }^{1}$, Nagalakshmi C.S ${ }^{2}$, Prashant $\mathbf{G}^{3}$, Rashmi BM ${ }^{4}$, Ganashree CP $^{5}$, Veluri Ganesh ${ }^{6}$ \\ ${ }^{1}$ Assistant Professor, Department of Biochemistry, Basaveshwara Medical College, Chitradurga. \\ ${ }^{2}$ Associate Professor, Department of Biochemistry, Akash Institute of Medical Sciences and Research Centre, Devanahalli, Bangalore. \\ ${ }^{3}$ Professor, Department of Medicine, ${ }^{4}$ Assistant Professor, Department of Community Medicine, ${ }^{5}$ Associate Professor, \\ Department of Physiology, ${ }^{6}$ Tutor, Department of Biochemistry, Basaveshwara Medical College, Chitradurga.
}

\begin{abstract}
Introduction: Dyslipidemia is the most common metabolic derangement of diabetes mellitus and is responsible for complications. Long term exposure to high glucose and fatty acid levels can damage the cells by process of oxidative stress. The oxidative stress in (DM) is also aggravated by impairment of the oxidant and antioxidant balance which leads to generation of excess free radicals. These induce membrane lipid peroxidation and generate lipid peroxides, which in turn disintegrates quickly to form reactive carbon compounds. Malondialdehyde (MDA) is such an important reactive carbon compound which is used commonly as an indicator of lipid peroxidation, and it has become an important oxidative stress marker. Majority of previous studies used serum MDA for assessing oxidative stress, but it is less reliable compared to erythrocyte MDA, as they are known to be prone for more oxidative reactions.
\end{abstract}

Aim and Objectives: The present study was taken to up to evaluate erythrocyte MDA levels among type 2 diabetic patients as compared to controls and to investigate correlation of erythrocyte MDA levels with lipid profile parameters in type 2 diabetic patients.

Materials and methods: Study included 60 type 2 diabetic patients and 60 healthy controls. Erythrocyte MDA levels and lipid profile parameters was analyzed among the patients and healthy controls by standard laboratory methods.

Results: Serum MDA levels and erythrocyte MDA levels werevery significantly higher in type 2 diabetics as compared to controls $(\mathrm{p}<0.001)$. Also, significant positive correlation was obtained between erythrocyte MDA levels and lipid profile parameters other than HDL. Instead, HDL cholesterol exhibited a significant negative correlation $(\mathrm{p}<0.001)$.

Conclusion: Based on our study results, it was concluded that poor glycemic control and dyslipidemia in type 2 diabetes mellitus are associated with increased erythrocyte MDA, which is an early marker of lipid peroxidation. This erythrocyte MDA can be used as a potential prognostic marker in prediction of oxidative stress. It has more value when used in diabetic patients with complications, which helps in identifying onset of complications early, thereby improving the quality of life and reducing disease progression.

Keywords: Erythrocyte malondialdehyde, Lipid profile, Type 2 diabetes, Oxidative stress, Lipid peroxidation

\section{INTRODUCTION:}

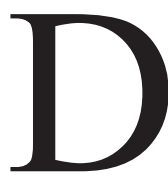
iabetes mellitus (DM) is a multisystem disorder, resulting from hyperglycemia, caused either due to absolute or relative deficiency of insulin, decreased glucose utilization and increased glucose production. It is a major global health problem, affecting almost all age groups. Diabetes prevalence is predicted to exponentially increase globally from 171 million to

Address for correspondence:

Dr. Nagalakshmi.C.S, Associate Professor, Department of Biochemistry, Akash Institute of Medical Sciences \& Research Centre,

Devanahalli - 562110. Email:nagu.smile@gmail.com Mobile no:09241852058

DOI : http://dx.doi.org/10.7324/NJBMS.2018.8302 
366 million in over three decades, with its maximum impact in India. ${ }^{1}$ Probably, by 2030, it may even affect nearly 79.4 million individuals in India. Dyslipidemia is the most common metabolic derangement seen in diabetes mellitus and it is responsible for both micro - and macrovascular complications, which finally results in organ and tissue damage. ${ }^{2}$ Long term exposure to high glucose and fatty acid levels can eventually cause cellular damage due to oxidative stress. ${ }^{3}$ The oxidative stress in diabetes is greatly increased by prolonged exposure to hyperglycemia and an impairment of oxidant - antioxidant balance. Lipids are among the primary targets of oxidative stress. ${ }^{4}$ Excess production of these free radicals, together with impairment of antioxidant defence mechanism leads to increased oxidative stress in diabetes, which induces membrane lipid peroxidation, thus generating fatty acid peroxides and causing cellular dysfunction. ${ }^{5}$ These peroxides are responsible for generation of reactive carbon compounds. On such compound, malondialdehyde (MDA) is an important reactive carbon compound which is used commonly as an indicator of lipid peroxidation, and has become one of the widely analysed oxidative stress marker, and especially so of lipids. ${ }^{6}$

This oxidative stress is responsible for pathogenesis of diabetes and its complications including cardiovascular disease. Thus, in greater number of previous studies, researchers have analyzed the oxidative status by using serum MDA level. ${ }^{7}$ However, serum MDA levels are less reliable when compared to erythrocyte MDA levels. Thorough search of literature shows that oxidative status is reflected more accurately by erythrocyte MDA levels rather than serum MDA levels since the latter is more susceptible for oxidative stress as well as erythrocyte membrane is rich in polyunsaturated fatty acids, so it is earliest to undergo lipid peroxidation compare to other cellular membrane. ${ }^{8}$ In this background, the present study was taken up to evaluate erythrocyte MDA levels among type 2 diabetic patients as compared to controls and to investigate correlation of erythrocyte MDA levels with lipid profile in type 2 diabetic patients.

\section{MATERIALS AND METHODS:}

In this cross-sectional study, about 60 type 2 diabetes mellitus patients, diagnosed as per American Diabetes Association (ADA) ${ }^{9}$ criteria attending Basaveshwara Medical College Hospital \& Research Centre, Chitradurga in the year 2017 were enrolled for the current study. Patients with acute \& chronic inflammatory diseases, thyroid disorder, liver disease, macro vascular complications such as cardiovascular / cerebrovascular / peripheral vascular diseases, type-1 diabetes, hypertension, those with history of smoking / alcoholism and patients receiving antioxidant therapies were carefully excluded from the study. 60 healthy individuals were included as controls. All these subjects were explained about the study and were included after obtaining informed consent. The study was also approved by Institutional ethics committee.

\section{Sample Collection:}

$4 \mathrm{ml}$ of fasting venous blood sample was collected from all subjects into two tubes: $1 \mathrm{ml}$ into a vacutainer containing anti glycolytic and anticoagulant, and $3 \mathrm{ml}$ into a plain vacutainer. Plasma samples were separated immediately and plain samples were allowed to clot and separated by centrifugation at $3000 \mathrm{rpm}$ for $15 \mathrm{~min}$. The 
Basavaraj Savadi, et.al : Erythrocyte MDA and lipids in diabetes

separated samples were transferred into appropriately labelled aliquots and biochemical analysis was done. Fasting blood glucose, total cholesterol, triglycerides, HDL-C and glycated haemoglobin were measured using laboratory standard methods. LDL-C was calculated by Friedwald's formula. Serum MDA and erythrocyte MDA levels (Ohkawa et al) ${ }^{10}$ were analyzed using thiobarbituric acid method.

\section{Statistical Analysis:}

The data was analysed and summarized descriptively. For continuous variables, mean and standard deviation were used. For categorical data, the number and percentage were used.

The difference in erythrocyte MDA level between patients and controls was analysed using unpaired Student's t-test (2-tailed). The correlation between erythrocyte MDA levels and lipid profile parameters was studied using Pearson correlation analysis. Data was compiled in Microsoft excel spread sheets and analyzed using SPSS for windows version 16.0. Statistically significant association was considered based on $\mathrm{p}$ value of $<0.05$

\section{RESULTS:}

Patient characteristics are shown in Table 1. The mean erythrocyte MDA levels of 60 diabetic patients studied was $4.94 \pm 0.21 \mathrm{nmol} / \mathrm{mg}$ and mean serum MDA was $6.7 \pm 0.56 \mathrm{nmol} / \mathrm{ml}$. Serum MDA levels and Erythrocyte MDA levels were significantly higher among type 2 diabetes mellitus patients as compared to serum MDA levels $(2.9 \pm$ $0.16 \mathrm{nmol} / \mathrm{ml})$ and erythrocyte MDA levels $(3.28 \pm$ $0.19 \mathrm{nmol} / \mathrm{mg}$ ) of controls $(\mathrm{p}<0.001)$.
Table 1.Patients characteristics and biochemical parameters studied in healthy controls and type 2 diabetes mellitus patients

\begin{tabular}{|c|c|c|c||}
\hline Parameter & $\begin{array}{c}\text { Controls (n=60) } \\
\text { (Mean } \pm \text { SD) }\end{array}$ & $\begin{array}{c}\text { T2DM (n=60) } \\
(\text { Mean } \pm \text { SD) }\end{array}$ & p value \\
\hline Age (years) & $49.13 \pm 8.20$ & $50.27 \pm 8.66$ & 0.226 \\
\hline BMI (kg/m $)$ & $24.22 \pm 2.94$ & $25.14 \pm 3.45$ & 0.366 \\
\hline FBG (mg/dl) & $98.93 \pm 11.52$ & $221.13 \pm 44.59$ & $<0.001$ \\
\hline Serum Cholesterol (mg/dl) & $162.94 \pm 37.43$ & $250.94 \pm 31.91$ & $<0.05$ \\
\hline Serum Triglyceride & $127.08 \pm 44.24$ & $228.12 \pm 40.07$ & $<0.001$ \\
\hline (mg/dl) & & & $<0.001$ \\
\hline Serum HDL (mg/dl) & $45.60 \pm 4.05$ & $32.50 \pm 3.265$ & $<0.05$ \\
\hline Serum LDL (mg/dl) & $99.36 \pm 34.49$ & $167.42 \pm 29.22$ & $<0.001$ \\
\hline HbA $\mathbf{1 C}$ (\%) & $5.84 \pm 0.28$ & $8.98 \pm 0.96$ & $<0.001$ \\
\hline Serum MDA (nmol/ml) & $2.9 \pm 0.16$ & $6.7 \pm 0.56$ & $<0.001$ \\
\hline Erythrocyte MDA & $3.28 \pm 0.19$ & $4.94 \pm 0.21$ & \\
\hline (nmol/mg of protein) & & & \\
\hline
\end{tabular}

Independent student $t$ test applied. There was also significant difference in mean values of lipid profile parameters and $\mathrm{HbA1C}$ levels in cases compared to controls.

Table 2. Correlation between erythrocyte MDA level and lipid profile parameters in type 2 diabetes mellitus patients

\begin{tabular}{|c|c|c|}
\hline Parameter & r value & p value \\
\hline Erythrocyte MDA and FBG & 0.756 & $<0.001$ \\
\hline Erythrocyte MDA and HbA1C & 0.816 & $<0.001$ \\
\hline $\begin{array}{c}\text { Erythrocyte MDA and Total cholesterol } \\
\text { Erythrocyte MDA and Serum }\end{array}$ & 0.543 & $<0.05$ \\
\hline Triglyceride & 0.453 & $<0.001$ \\
\hline $\begin{array}{c}\text { Erythrocyte MDA and HDL cholesterol } \\
\text { Erythrocyte MDA and LDL cholesterol }\end{array}$ & -0.502 & $<0.001$ \\
\hline
\end{tabular}

Data summarized in Table 2 concludes that there is significant positive correlation between erythrocyte MDA levels and FBG, HbA1C, Total cholesterol, Triglyceride and LDL-C levels, but a significant negative correlation between 
erythrocyte MDA level and HDL-C levels. Erythrocyte MDA shows a significant and positive correlation with FBG, HbA1C, Total Cholesterol, serum triglycerides and LDL-C and a significant negative correlation with HDL-C levels (Pearson correlation analysis).

\section{DISCUSSION:}

Diabetes mellitus is a metabolic disorder characterised by relative or absolute deficiency of insulin leading to persistent hyperglycemia. In the present study, serum levels of fasting blood glucose and glycated haemoglobin were found to be significantly higher among cases as compared to controls. These findings are in accordance with previous studies by Verma $\mathrm{M}^{11}$ and Srivastan $\mathrm{R}^{12}$ and it is well documented that MDA is a stable end product of free radicals induced by lipid peroxidation. Thus MDA provides an assessment of free radical induced tissue damage. Two important consequences of hyperglycaemia in diabetes mellitus are oxidative stress and formation of advanced glycation end products (AGEs). In diabetic patients, a major factor that is responsible for enhanced free radical generation is increased glucose auto-oxidation, polyol pathway, nonenzymatic and auto-oxidative glycosylation of proteins. $^{13}$

In uncontrolled diabetes, the activity of pentose phosphate pathway is increased leading to excessive production of NADPH, which in turn promotes lipid peroxidation in presence of cytochrome P450. Lipid peroxidation in $\mathrm{RBC}$ membrane is increased by oxyhemoglobin in erythrocytes which acts like Cytochrome. This increased oxidative stress results in damage to erythrocyte membrane proteins which is a consequence of hyperglycemia. Lipid peroxidation damages are responsible for the changes in membrane properties of RBC. ${ }^{14}$

In this study, there is a significant increase in erythrocyte MDA levels in type 2 diabetic patients as compared to controls and it correlates positively with HbA1C which is statistically significant. Studies conducted by Srivastan $\mathrm{R}$ et $\mathrm{al}^{12}$, Mousa SA et $\mathrm{al}^{15}$ have shown similar findings of increased erythrocyte MDA levels in type 2 diabetics as compared to controls. Elevated erythrocyte MDA levels among diabetics are thought to be due to persistent hyperglycemia and an imbalance in oxidant-antioxidant levels, causing oxidative stress. Using correlation analysis, it was found that erythrocyte MDA levels had significant positive correlation with FBG, Glycated haemoglobin, Total cholesterol, Triglycerides and LDL cholesterol (Table-2) and significant negative correlation with HDL cholesterol levels. This study also shows there is significant increase in serum MDA levels in type 2 daibetic patients as compared to controls which indicates the oxidative stress. Similar results are documented in the studies conducted by Arora $\mathrm{M}$ et al, Rani AJ et al and Ikekpeazu EJ et al. ${ }^{16,17,18}$

Type 2 diabetes mellitus is a chronic progressive metabolic disorder characterised by hyperglycaemia and dyslipidemia, which makes cells more susceptible for lipid peroxidation and inflammation due to oxidative stress and it is thought to play a major role in the pathogenesis of diabetes and its complications. ${ }^{19}$

\section{CONCLUSION:}

The results of this study and previous works provide ample evidence that poor glycemic control and dyslipidemia in type 2 diabetes mellitus are 
associated with increased erythrocyte MDA, which is an earlier marker of lipid peroxidation than serum MDA because these are more susceptibility to oxidative attack by lipid peroxides. Erythrocyte MDA levels will be elevated prior to systemic oxidative stress marker, serum MDA. Hence erythrocyte MDA can be used as a potential prognostic marker in predicting the oxidative stress earlier than the serum MDA. It has more value when used in diabetic patients with complications such as hypertension, ischemic heart disease, lipid abnormalities and uncontrolled diabetes and helps in identifying the onset of complications at an early stage, thereby improving the quality of life and reducing disease progression in type 2 diabetic patients.

\section{LIMITATIONS:}

Further studies on larger population and geographic areas needed to establish erythrocyte MDA as a potential prognostic marker for oxidative stress.

\section{REFERENCES:}

1. Stumvoll M, Goldstein BJ, van Haften TW. Type 2 diabetes: Principles of pathogenesis and therapy. Lancet 2005; 365: $1333-46$.

2. King H, Aubert RE, Herman WH. Global burden of diabetes 1995-2025: Prevalence, numerical estimates, and projections. Diabetes Care 1998; 21: 1414-31.

3. Nakhjavani M, Esteghamati A, Nowroozi S, Asgarani F, Rashidi A, Khalilzadeh O. Type 2 diabetes mellitus duration: an independent predictor of serum malondialdehyde levels. Singapore Medical Journal2011; 51(7): 582.

4. Ramakrishna V, Jailkhani R. Oxidative stress in non-insulin-dependent diabetes mellitus (NIDDM) patients. ActaDiabetologica 2008; 45: 41-6.

5. Sanocka D, Kurpisz M. Reactive oxygen species in sperm cells. Reproductive Biology and Endocrinology 2004; 224:2-12.

6. Placer ZA, Lind L, Cushmann M, Johnson BC. Estimation of product of lipid peroxidation (MDA) in biological systems. Analytical Biochemistry 1966; 16: 359-64.

7. Kose K, Yazici C, Cambay N, Ascioglu O, Dogan P. Lipid peroxidation and erythrocyte antioxidant enzymes in patients with Behcet's disease. The Tohoku Journal of Experimental Medicine 2002; 197: 9-16.

8. Cross CE, Halliwell B, Borish ET, Pryor WA, Ames BN, Saul RL, et al. Oxygen radicals and human disease. Annals of Internal Medicine 1987; 107: 526-545.

9. Classification and Diagnosis of Diabetes, American Diabetes Association. Diabetes Care 2016; 39(Supp1.1): S13-S22.

10. OhkawaH,OhishiN, Yagi K et al. Assay for lipid peroxides in animal tissues by thiobarbituric acid reaction. Annals of Biochemistry 1979 Jun ;95(2):351-8.

11. Verma M, Paneis S, Badi P, Raman PG. Effect of increasing duration of diabetes mellitus type 2 on Glycated haemoglobin and insulin sensitivity. Indian Journal of Clinical Biochemistry 2006; 21(1): 142-46.

12. Srivastan R, Das S, Gadde R, Manoj kumar K, Taduri S, Rao $\mathrm{N}$ et al. antioxidant and lipid peroxidation status in diabetic patients with and without complications. Archives of Iranian Medicine 2009; 12: 121-7. 
13. Noberasco G, Odetti P, Boeri D, Maiello M, Adezati L. Malondialdehyde (MDA) level in diabetic subjects. Relationship with blood glucose and glycosylated hemoglobin. Biomedicine and Pharmacotherapy 1991; 45: 193-6.

14. Sharma M, Arora M, Imran M, Sudeep K, Anju M, Sandeep SS. Correlation of Glycated Hemoglobin with Oxidative Stress and Erythrocyte Fragility in Type-2 Diabetes Mellitus. International Journal of Contemporary Medical Research 2017; 4: 1909-11.

15. Mousa SA. Oxidative stress in diabetes mellitus. Romanian Journal of Biophysics 2008; 18(2): 222-36.

16. Arora M, Mahat RK, Kumar S, Tyagi S, Batra J. Oxidative stress and its relation to glycemic control in patients of type 2 diabetes mellitus. International Journal of Medical Science and Public Health 2016; 5: 1173-1177.

17. Rani AJ, Mythili SV. Study of oxidative stress and its relation to glycemic control in type 2 diabetes mellitus. Research Journal of Pharmaceutical, Biological and Chemical Sciences 2014; 5: 589-92.

18. Ikekpeazu EJ, Neboh EE, Ejezie FE, Ibegbu MD, Ike IE. Oxidative stress and glycaemic control in type 2 diabetic patients in Enugu, South-East Nigeria. Annals of Medical and Health Sciences Research 2011; 1: 123-8.

19. G. D. Bhambhani GD, Bhambhani RG1, Nilesh C. Lipid profile of patients with diabetes mellitus: a cross sectional study, International Journal of Research in Medical Sciences 2015 Nov; 3(11): 3292-3295. 